\title{
Effects of microRNA-27a Targeting Smad1 on Intervertebral Disc Degeneration and Biological Characteristics of Nucleus Pulposus Cells
}

\section{Heng-Tao Tang ( $\nabla$ httang0@163.com )}

Department of orthopaedics, The First Affiliated Hospital of Zhengzhou University, 450052, Henan Province,China https://orcid.org/0000-0002-3478-5518

\section{Song Zhang}

The First Affiliated Hospital of Zhengzhou University

\section{Xin-Chang Lu}

The First Affiliated Hospital of Zhengzhou University

\section{Tong-Yu Geng}

The First People's Hospital of Shangqiu

\section{Research article}

Keywords: miR-27a, intervertebral disc degeneration, Smad1, nucleus pulposus cells, extracellular matrix

Posted Date: November 2nd, 2020

DOl: https://doi.org/10.21203/rs.3.rs-99036/v1

License: (9) This work is licensed under a Creative Commons Attribution 4.0 International License. Read Full License 


\section{Abstract}

Objective: The present study aimed to analyze the expression of microRNA-27a (miR-27a) in intervertebral disc degeneration (IDD) and its effect on the biological characteristics of nucleus pulposus (NP) cells.

Methods: An IDD rat model was established, and the expression of miR-27a and Smad1 in the intervertebral disc tissue was detected. An oxygen and glucose deprivation (OGD) NP cell model was established to simulate the IDD microenvironment, and the effects of downregulated miR-27a on the proliferation, apoptosis, inflammatory response, and extracellular matrix (ECM) proteins of OGD-NP cells were analyzed. The target relationship of miR-27a and Smad1 was verified by luciferase reporter assays, and siRNA-Smad1 was transfected to reverse the experiment.

Results: The level of miR-27a in the IDD model group was significantly increased, whereas that of Smad1 was decreased compared with the sham group $(P<0.05)$. Inhibition of miR-27a improved cell proliferation, and inhibited apoptosis, degradation of the ECM, and inflammatory response of OGD-NP cells compared with the OGD group $(P<0.05)$. The results of the double luciferase reporter assays indicated that Smad1 was the target gene of miR-27a. Smad1 silencing reversed the increase in ECM proteins induced by inhibition of miR-27a; However, it did not affect cell proliferation and apoptosis.

Conclusion: The expression levels of miR-27a were upregulated in IDD and it may be involved in the progression of IDD by promoting the apoptosis of NP cells and ECM degradation by targeting Smad1.

\section{Introduction}

The intervertebral disc (IVD), the largest non-vascular organ in the human body, is composed of the nucleus pulposus (NP), annulus fibrosus (AF), and cartilaginous endplate [1]. NP cells play a key role in maintaining the biological characteristics and tensile strength of the IVD by synthesizing and secreting collagen II and producing extracellular matrix (ECM). Intervertebral disc degeneration (IDD) is the pathological basis of a series of spinal degenerative diseases. At present, it is thought that the pathogenesis of IDD is mainly related to aging, mechanical stress, degradation of the ECM marked by progressive loss of aggrecan and collagen II, and genetic factors[2]. Among them, ECM synthesis and degradation caused by the abnormal proliferation and apoptosis of NP cells are considered to be key factors in the pathogenesis of $\operatorname{IDD}[3,4]$.

Numerous studies have shown that a variety of miRNAs play important roles in the degradation of the ECM and regulation of proliferation and apoptosis of NP cells[5]. MicroRNA-27a (miR-27a) is abnormally expressed in IDD tissues and involved in the regulation of NP cell proliferation and inflammatory response [6, 7]. However, whether it participates in the development of IDD and abnormal regulation of NP cells, as well as its mechanism of influence on the ECM remains unclear. Our previous studies showed that miR-27a may participate in the progression of IDD by regulating the degradation of the ECM. Bioinformatics analysis showed that Smad1 may be the major target protein mediating this mechanism. The Smad family includes the main proteins mediating the transforming growth factor- $\beta$ (TGF- $\beta$ ) 
signaling pathway. According to their functions, they are termed R-Smad, co-Smad, and i-Smad. R-Smad includes Smad $2 / 3$ activated by TGF- $\beta$ and Smad I/5/8/9 activated by bone morphogenetic protein (BMP)[8]. Studies have confirmed that both TGF- $\beta / S m a d 2 / 3$ and BMP/Smad1 signaling pathways play key roles in cartilage development and repairment, as well as ECM synthesis and degradation[9]. Thus, we hypothesized that miR-27a may affect the development of IDD by targeting and regulating Smad1. The aim of this study was to investigate the mechanism of miR-27a in the occurrence and development of IDD, and analyze whether Smad1 is the target protein mediating this mechanism.

\section{Materials And Methods}

\subsection{Animal model construction}

Twenty male Sprague-Dawley rats, aged 6-8 weeks, (purchased from the Experimental Animal Center of ; quarantine license No.: SCXK(Yu) 2017-0001) were randomly divided into the sham group $(n=10)$ and model group $(n=10)$. The rats were anesthetized by intrap

eritoneal injection of $5 \%$ chloral hydrate. The animals were in the supine position, and a right paracentral incision was performed. The skin and subcutaneous tissue were successively incised. Subsequently, the abdominal cavity was opened, and the posterior peritoneum was cut to expose the upper and lower cartilage endplates of L5/6 IVD. Next, the above IVD segments were punctured with a 21-G micropuncture needle, which was inserted parallel to the cartilage endplate; the depth of needling was the whole layer of the AF. After the operation, the subcutaneous fascia and skin were successively sutured. In the sham group, the skin tissue was only cut to expose the IVD and directly sutured. All rats were injected with penicillin $\left(8 \times 10^{4} \mathrm{U} /\right.$ day) for 3 days to prevent infection.

\subsection{Pathological observation of hematoxylin-eosin (HE) staining}

At 8 weeks after the modeling operation, rats were euthanized by intraperitoneal injection of excessive pentobarbital $(60 \mathrm{mg} / \mathrm{kg})$. The IVD tissues of the 5 th and 6 th lumbar vertebrae were rapidly and completely removed. The bloodstains were washed with sterile normal saline, and parts of the tissues were stored in liquid nitrogen. The remaining parts were fixed in paraformaldehyde for $24 \mathrm{~h}$, decalcified with $10 \%$ ethylenediaminetetraacetic acid for $6 \mathrm{~h}$, embedded in paraffin, and continuously sectioned (thickness: $4 \mu \mathrm{m}$ ). The slices were dewaxed in xylene for $10 \mathrm{~min}$, and rehydrated with gradient alcohol. Subsequently, the samples were placed in hematoxylin dye solution for $15 \mathrm{~min}$, dyed in $1 \%$ eosin solution for 10 min, washed, dehydrated, made transparent using xylene, and sealed after air drying. The pathological sections were observed under a light microscope.

\subsection{Immunohistochemical experiment}


After dewaxing with xylene and rehydration with gradient ethanol, rabbit anti-rat Smad1 primary antibody (ab80255, 1:100; Abcam) was added to the tissues according to required proportion. The reaction was performed for $30 \mathrm{~min}$ at room temperature. After washing with phosphate-buffered saline (PBS), horseradish peroxidase-labeled goat anti-rabbit secondary antibody (ab6721, 1:1,000; Abcam) was added. The tissues were incubated for $30 \mathrm{~min}$ at $37^{\circ} \mathrm{C}$, and washed with PBS for $3 \times 5 \mathrm{~min}$. Subsequently, $3,3^{\prime}$-diaminobenzidine was added, dyed, dried, and sealed. Semi-quantitative analysis was used to evaluate the degree of positive expression: the positive staining intensity score was 1-3 points from light to heavy, while the 0 point indicated no staining. The proportions of positive cells (i.e., $0-5 \%, 6-25 \%, 26-$ $50 \%, 51-75 \%$, and $76-100 \%$ ) were scored using $0-4$ points, respectively. The final result was the sum of the staining score and positive cell proportion score.

\subsection{Isolation and culture of NP cells}

Following resuscitation, the rat lumbar IVD tissue of the sham group was stored in liquid nitrogen, cut to obtain the complete NP tissue, and placed in a Petri dish containing PBS. After being cut to the size of 1 $\mathrm{mm}^{3}$, the tissue was placed in $0.1 \%$ type Il collagenase ( $\times 5$ volume), digested for $4 \mathrm{~h}$ at $37^{\circ} \mathrm{C}$, and centrifuged at 1,000 rpm for $5 \mathrm{~min}$. The supernatant was discarded, and the cells were washed once with PBS. Next, the cells were resuspended and counted in complete medium (DMEM/F12 medium containing $10 \%$ fetal bovine serum (Gibco) and 1\% penicillin-streptomycin). Cells were seeded in a $25-\mathrm{cm}$ culture flasks at a density of $1 \times 10^{6} \mathrm{cells} / \mathrm{ml}$, and placed in an incubator at $37^{\circ} \mathrm{C}, 5 \% \mathrm{CO}_{2}$, and saturated humidity environment. The isolated NP cells were divided into the NPC group and the oxygen and glucose deprivation (OGD) group. The cells in the NPC group were cultured in DMEM medium containing $10 \%$ fetal bovine serum (Gibco). According to the growth of cells, the medium was changed once every 2-3 days. When the cells reached $80-90 \%$ confluence, they were digested with trypsin and subcultured. In the OGD group, after the cells adhered to the flask wall, the well-grown cell culture medium was replaced with serum-free DMEM medium. The cells were subsequently cultured in a $1 \% \mathrm{O}_{2}+94 \% \mathrm{~N}_{2}+5 \% \mathrm{CO}_{2}$ gas component incubator for $48 \mathrm{~h}$ to simulate the micro-environment of IVD degeneration in vivo.

\subsection{Cell grouping and transfection}

The OGD cells were divided into the OGD, miR-27a inhibitor, inhibitor-negative control (inhibitor-NC), and miR-27a inhibitor+si-Smad1 groups. The last three groups were transfected with a miR-27a inhibitor plasmid (Sangon, Shanghai, China), negative control plasmid, and miR-27a inhibitor +Smad1 interference plasmid (siRNA-Smad1), respectively. For the transfection method, cells in the logarithmic phase were seeded in a six-well plate. When the cells reached $70 \%$ confluence, they were transfected using Lipofectamine 2000 (Invitrogen) according to the instructions provided by the manufacturer.

\subsection{Quantitative reverse transcription-polymerase chain reaction (qRT-PCR)}


Trizol (Invitrogen) was used to extract total RNA from tissues or cells ( $24 \mathrm{~h}$ after transfection), which was ground into powder in liquid nitrogen. A reverse transcription kit (Takara) was used for the reverse transcription of RNA into CDNA. The forward and reverse primer sequences of miR-27a used were $5^{\prime}-$ CTGTGGAGCAGGGCTTAG-3' and 5'-TAGCCACTGTGAACAC GACT-3' respectively, and those of U6 were 5'CTCGCTTCGGCAGCACA-3' and 5'-AACGCTTCACGAATTTGCGT-3' respectively. SYBR Green (Takara) was used for qRT-PCR. The reaction conditions were as follows: $95^{\circ} \mathrm{C}$ for $10 \mathrm{~min}, 95^{\circ} \mathrm{C}$ for $15 \mathrm{~s}$, and $60^{\circ} \mathrm{C}$ for $15 \mathrm{~s}$, for a total of 40 cycles. Each sample was analyzed thrice. U6 was used as an internal reference. The relative expression was calculated using the $2^{-\Delta \Delta C t}$ method.

\subsection{Western blotting}

A cell total protein extraction kit(Solarbio, Beijing) was used to extract the total protein. The bicinchoninic acid(BCA) method was used to determine the protein concentration. Sodium dodecyl sulfatepolyacrylamide gel electrophoresis (SDS-PAGE) was performed to transfer the proteins to a polyvinylidene fluoride membrane, which was blocked in $5 \%$ milk. Subsequently, the membrane was incubated with primary antibodies overnight at $4^{\circ} \mathrm{C}$, namely rabbit anti-rat Smad1 (ab80255, 1:1,000; Abcam), matrix metalloproteinase (MMP13; ab39012, 1:3,000; Abcam), aggrecan (ab36861, 1:1000; Abcam), and mouse anti-rat collagen II (ab185430, 1:200; Abcam). After washing the membrane, it was incubated with horseradish peroxidase-labeled goat anti-rabbit IgG secondary antibody (ab7090, 1:5,000; Abcam) or goat anti-mouse IgG secondary antibody (ab97040, 1:5,000; Abcam) at room temperature for $1 \mathrm{~h}$. Enhanced chemiluminescence was used to visualize the proteins. The software ImageJ was used for image analysis. Glyceraldehyde-3-phosphate dehydrogenase or $\beta$-actin were used as internal control to calculate the relative protein expression.

\subsection{Enzyme-linked immunosorbent assay (ELISA)}

At $24 \mathrm{~h}$ after transfection,ELISA kits were used to detected the levels of interleukin-6 (IL-6) and tumor necrosis factor- $\mathrm{a}$ (TNF-a) in the cells. The optical density was measured at $450 \mathrm{~nm}$ with an microplate reader (Thermo Fisher) according to the instructions provided by the manufacturer.

\subsection{Cell Counting Kit-8 (CCK-8) assay for the detection of cell proliferation}

At $24 \mathrm{~h}$ after transfection, the cells were seeded in 96-well plates with five multiple pores in each group. After the cells adhered to the plate wall, the cell viability was detected using the CCK-8 assay for $24 \mathrm{~h}, 48$ $\mathrm{h}$, and $72 \mathrm{~h}$, respectively. At $2 \mathrm{~h}$ prior to detection, $10 \mu \mathrm{l}$ CCK-8 reagent was added into each well, and the culture was continued for $2 \mathrm{~h}$ in the incubator. The absorbance of each pore sample was detected at 450 $\mathrm{nm}$ through multifunctional ELISA. The cell viability was calculated using normal cells as control. 


\subsection{Flow cytometry for the detection of apoptosis and cell cycle analysis}

At $48 \mathrm{~h}$ after transfection, the transfected cells were washed with PBS, and 70\% precooled ethanol was added to fix the cells overnight at $4^{\circ} \mathrm{C}$. After another wash with PBS, $2 \mu \mathrm{l}$ RNase $\mathrm{A}(0.25 \mathrm{mg} / \mathrm{ml})$ and 500 $\mu \mathrm{l}$ propidium iodide dye solution $(50 \mu \mathrm{g} / \mathrm{ml})$ were added in the dark at room temperature for $30 \mathrm{~min}$. The cell cycle in each group was determined.

At $48 \mathrm{~h}$ after transfection, the transfected cells were washed with PBS, and a cell suspension was prepared. The cell density was adjusted to $1 \times 10^{5} \mathrm{cell} / \mathrm{ml}$. Annexin V-fluorescein isothiocyanate $(5 \mu \mathrm{l})$ was added to $100 \mu \mathrm{l}$ of cells for $15 \mathrm{~min}$ at room temperature. Propidium iodide was added 5 min prior to the operation, and the degree of apoptosis was detected using flow cytometry(CytoFLEX).

\subsection{Luciferase reporter assay}

According to the sequence of the Smad1 gene in the plasmid pmirGLO and on PubMed, the wild-type (WT) and mutant (Mut) primers with Sacl and Xhol on both ends were designed to amplify the WT fragment (5'-ATAATACTTGACCTCTGTGAC-3') and Mut fragment (5'-ATAATTGAATAGGTGACACTT-3') of the Smad1 gene 3'untranslated region (3'UTR), and construct the WT plasmid pmirGLO-Smad1-3'UTR-WT and pmirGLO-Smad1-3'UTR-Mut, respectively. NPC cells were seeded in 24-well plates, cultured for $24 \mathrm{~h}$. After the cells adhered to the wall, the cells were co-transfected with the pmirGLO-Smad1-3'UTR-WT plasmid or pmirGLO-Smad1-3'UTR-Mut plasmid and miR-27a mimic or miR-NC. At $48 \mathrm{~h}$ after transfection, the luciferase activity was detected using a double luciferase reporter assay kit (Promega).

\subsection{Statistical analysis}

All data were analyzed using SPSS version 19.0 (IBM Corp., Armonk, NY, USA. The independent sample $t$ test was used to compare the measurement data between the two groups. One-way analysis of variance and the least significant difference- $t$ test were used for comparison and pairwise comparison among the groups, respectively. $P<0.05$ denotes statistically significant difference.

\section{Results}

\subsection{Pathological observation of IDD tissue by HE staining}

HE staining showed that, compared with the sham group, IVD tissue in the model group presented the following: AF rupture, tissue texture disorder, inflammatory infiltration, reduction in the number of NP cells to some degree, and apoptosis-like changes in some cells (Figure 1A). Usually, IDD begins from the destruction and degradation of NP tissue[2], which mainly consists of NP cells, collagen II, and aggrecan [10]. Collagen II and aggrecan are the main markers of ECM degradation in the process of IDD. In the 
present study, western blotting analysis indicated that the expression of collagen II and aggrecan was significantly lower in the model group compared with the sham group $(P<0.05)$ (Figure 1B). These results suggested that the rat model of IDD was successfully established.

\subsection{Expression of miR-27a and Smad1 in the IDD model}

Previous studies have shown that miR-27a was differentially expressed in IDD tissues [6]. Therefore, the expression of mir-27a in IDD rats was detected by qRT-PCR. The results showed that miR-27a was significantly upregulated in the model group compared with the sham group (Figure 2A). Our previous study demonstrated that Smad1 may be one of the targets of miR-27a in the IVD. Other studies have also shown that Smad1 plays an important role in tissue fibrosis and chondrocyte apoptosis in arthritis. In the present study, immunohistochemical analysis indicated that Smad1 was mainly expressed in the cytoplasm of NP cells. The degree of positivity was significantly lower in the model group than the sham group ( $P<0.05$, Figure $2 \mathrm{~B}$ ), which was consistent with the results of the western blotting analysis (Figure 2C). We analyzed the correlation of Smad1 and miR-27a expression, and the results indicated that they were significantly negatively correlated $(K<0, P<0.05)$ (Figure 2D).

\subsection{Expression of miR-27a and Smad1 in OGD NP cells}

We also investigated the mechanism of miR-27a in IDD. For this purpose, we constructed the OGD-NP cell model to simulate the microenvironment of NP cells in IDD tissues, and the cells were transfected with the miR-27a inhibitor to downregulate the expression of miR-27a. The cell morphology was observed under the light microscope:. The OGD group exhibited obvious adhesion and a vague outline, the boundary between the nucleus and cytoplasm was unclear, and a certain number of cells showed apoptosis compared with the NPC group. Following transfection with the miR-27a inhibitor, the pathological changes of cells were improved compared with the OGD group $(P<0.05)$ (Figure.3A). The levels of miR-27a in the OGD group were significantly higher, whereas the expression of Smad1 was significantly lower than that measured in the NPC group. Moreover, the expression levels of miR-27a were significantly decreased in the miR-27a inhibitor group, whereas those of Smad1 were significantly increased compared with the OGD group $(P<0.05)$ (Figures 3B, 3C).

\subsection{Effect of miR-27a on the proliferation and apoptosis of OGD-NP cells}

Abnormal proliferation and apoptosis of NP cells was the main cause of IDD. miR-27a is involved in the regulation of cell proliferation and apoptosis in many diseases, including malignant tumors [11]. CCK-8 assay demonstrated that the proliferative activity was significantly lower in the OGD group than the NPC group, while inhibition of the miR-27a levels significantly promoted the proliferative activity of OGD-NP cells $(P<0.05)$ (Figure 4A). Flow cytometry analysis indicated that the apoptosis rate and proportion of 
cells in the G1/G0 phase were higher in the OGD group than the NPC group. Furthermore, the proportion of $S$ phase cells in the miR-27a inhibitor group was higher than that of the OGD group. In contrast, the apoptosis rate in the miR-27a inhibitor group was lower than that of the OGD group $(P<0.05)$ (Figure 4B).

\subsection{Effect of miR-27a on inflammatory factors and the ECM}

Inflammation and ECM degradation are closely related to IDD. The inflammatory response is one of the main characteristics of the microenvironment in the degeneration IVD. IL- 6 and TNF- $a$ are the most typical inflammatory factors involved in this process. ELISA indicated that the levels of IL-6 and TNF- $a$ in the OGD group were significantly higher than those of the NPC group. In contrast, these levels in the miR-27a inhibitor group were significantly lower than those of the OGD group $(P<0.05)$ (Figure 5A). Additionally, the expression levels of collagen II and aggrecan in the OGD group were significantly lower than those of the NPC group. In contrast, these levels in the miR-27a inhibitor group were significantly higher than those of the OGD group $(P<0.05)$ (Figure 5B).

\section{6 miR-27a regulates OGD-NP cells by targeting Smad1}

The results above indicate that miR-27a was highly expressed, whereas Smad1 was lowly expressed in IDD tissues, and they were negatively correlated. To investigate whether miR-27a plays a role in IDD by targeting Smad1, we used TargetScan (http://www.targetscan.org/mamm_31/) to analyze the relationship between miR-27a and Smad1. The results showed that miR-27a could bind to the 3'UTR of Smad1 (Figure 6A). The results of the luciferase reporter gene assay also showed that the luciferase activity in the miR-27a mimic+Smad1-WT group was significantly lower than that of the miR-27a mimic+Smad1-Mut group $(P<0.05)$ (Figure 6A). Moreover, there was no difference in luciferase activity between the mimic-NC+Smad1-WT and mimic-NC+Smad1-Mut groups ( $P>0.05)$ (Figure 6A), which verified that miR-27a could regulate Smad1.

We sought to examine whether the targeted regulation of Smad1 by miR-27a was the mechanism involved in its effect on the biological characteristics of NP cells. Therefore, OGD-NP cells were cotransfected with the miR-27a inhibitor and siRNA-Smad1. The results showed that there were no significant changes in cell proliferation, apoptosis rate, and cell cycle between the siRNA-Smad1 group and miR-27a inhibitor group $(P<0.05)$ (Figures 6B-D). However, the levels of inflammatory factors IL-6 and TNF-a increased $(P<0.05)$ (Figure 6E), whereas those of collagen II and aggrecan proteins decreased in the siRNA-Smad1 group compared with the miR-27a inhibitor group $(P<0.05)$ (Figure 6F). These findings suggested that silencing Smad1 could reverse the changes in the levels of inflammatory factors and the ECM induced by inhibition of miR-27a. In conclusion, the data indicated that miR-27a may regulate the ECM and inflammatory factors of NP cells by targeting Smad1. However, this is not the mechanism through which miR-27a affects the proliferation and apoptosis of NP cells.

\section{Discussion}


IDD is the main cause of various spinal diseases. The IVD consists of $99 \%$ ECM proteins and $1 \%$ cells, most of which are NP cells. ECM degradation, abnormal apoptosis of NP cells, and inflammatory reaction are the main pathological processes of IDD. ECM proteins are mainly composed of collagen II and aggrecan, which are mainly secreted by NP cells [12]. Therefore, the balance between the growth and apoptosis of NP cells is key to the balance of the ECM and the development of IDD. Furthermore, the increased expression of inflammatory mediators, such as IL-1, IL-6, TNF-a, and TNF- $y$, is another characteristic of the IDD microenvironment [13]. Studies have shown that inflammatory cytokines (e.g., IL$1 \beta$ and TNF-a) could increase the expression of MMP13, reduce the expression of type II collagen and aggrecan, induce apoptosis of NP cells, and lead to progression of IDD [14, 15]. At present, targeted therapy has become a hot spot in research regarding treatment schemes for various diseases. Thus, investigation of the genes and signaling pathways involved in regulating the degradation of the ECM and NP cells function in IDD is of great importance.

miRNA inhibits the expression of target genes by binding to the 3'UTR of mRNA, and participates in the pathogenesis and progression of various diseases or biological processes, including degenerative disc diseases (e.g., IDD) [10]. Several studies have shown that miR-27a is involved in the progression of degenerative diseases (e.g., osteoarthritis) and orthopedic diseases. Cai et al. [16]showed that miR-27a promoted the progression of osteoarthritis by inducing autophagy and apoptosis of chondrocytes. Wang et al.[17] showed that inhibition of miR-27a levels could promote chondrocyte proliferation and inhibit ECM degradation, thus inhibiting the degradation of cartilage tissue. However, few studies investigated the correlation between miR-27a and IDD, and existing research studies have not reached a consistent conclusion. Liu et al.[7]was the first to propose that miR-27a was expressed in abnormally low levels in IDD tissues, and may inhibit apoptosis of NP cells by targeting PI3K/Akt signaling pathway to participate in the process of IDD. On the contrary, Cao et al.[6]showed that miR-27a was abnormally overexpressed in IDD tissues. Moreover, by inhibiting the expression of miR-27a in NP cells induced by lipopolysaccharide, the nuclear factor-kB/p38 signaling pathway was inhibited and the levels of IL-1 $1 \beta$ and IL- 6 inflammatory factors were significantly decreased. This evidence suggested that miR-27a acts as a promoter in IDD by activating the inflammatory factor pathway. The present study demonstrated that the expression levels of miR-27a were upregulated in the IVD of IDD rat and OGD-NP cell models, suggesting that miR-27a plays a positive role in IDD. Some studies have shown that miR-27a promotes the degradation of the ECM, thus preventing the occurrence of fibrosis. Liu et al.[18] showed that miR-27a could inhibit the activation of the Wnt/ $\beta$-catenin signaling pathway in the lung tissue of rats with pulmonary fibrosis, thus inhibiting the occurrence of tissue fibrosis, and secretion of collagen, and promoting the degradation of the ECM. This mechanism was also involved in myocardial fibrosis and detrusor fibrosis $[19,20]$. NP cells are the main cells secreting the ECM in IVD tissue. The abnormal proliferation and apoptosis of NP cells are the main reasons responsible for ECM deficiency and IDD. In most studies, miR-27a was regarded as a tumorinhibiting factor [21, 22]. Therefore, the upregulation of miR-27a can significantly inhibit cell proliferation and increase the rate of apoptosis. In the present study, inhibition of miR-27a improved cell proliferation, and inhibited apoptosis, the inflammatory response, and degradation of the ECM. These results revealed 
that miR-27a may participate in the progression of IDD by regulating apoptosis, the ECM, and inflammatory response.

Eight Smad proteins have been identified in mammals, all of which are downstream transcription regulators of the TGF- $\beta 1 / B M P$ signaling pathway [23]. Smad1 mainly mediates the activation of the BMP signaling pathway. In vitro studies have shown that TGF- $\beta$ /BMP pathway activity exerts a protective effect on articular cartilage[24]. It has been reported that TGF- $\beta$ is a protective factor against IDD. It can increase the synthesis of proteoglycan and collagen in degenerated human IVD cells, and reduce the levels of active MMPs in NP cells [25]. BMP/Smad1 plays a key role in the synthesis and degradation of the ECM in various degenerative diseases. Barruet et al.[26] showed that activation of the BMP4/Smad1 pathway could increase the expression of matrix fibrosis protein. Li et al.[27] showed that long-chain noncoding RNA TM1P3 was upregulated in osteoarthritis. Notably, downregulation of its expression could decrease the phosphorylation levels of Smad1 and promote ECM degradation, suggesting that Smad1 was negatively related to ECM degradation. Hodgkinson et al.[28] showed that Smad1 played an essential role in the differentiation of adipose-derived mesenchymal stem cells used for the treatment of IDD. Moreover, it promoted the production of aggrecan and type II collagen by cooperation with the extracellular signal-regulated kinase 1/2 pathway. Wang et al.[29] suggested that BMP7 could play an anti-ECM catabolism role in IDD by activating Smad1. The present study demonstrated that Smad1 expression was significantly low in IDD rats, and its expression levels were negatively correlated with miR27a. The expression of Smad1 was significantly increased after inhibition of miR-27a expression, and dual-luciferase reporter gene assay also confirmed their relationship. Co-transfection with the miR-27a inhibitor and siRNA-Smad1 reversed the up-regulation of collagen II and aggrecan induced by inhibition of miR-27a. However, the proliferative activity and apoptosis rate of NP cells did not change significantly. These findings suggested that the targeting of Smad1 by miR-27a was the main reason for the regulation of the ECM, rather than the mechanism of its influence on NP cells. However, the molecular mechanism of miR-27a involved in the pathogenesis of IDD by regulating the proliferation and apoptosis of NP cells warrants further investigation.

In summary, the present study indicated that the expression levels of miR-27a were upregulated in IDD, which may promote the development of IDD by promoting the apoptosis of NP cells, ECM degradation, and inflammatory response This evidence suggests that miR-27a may be a potential molecular target for IDD therapy. The targeted regulation of Smad1 is the main mechanism affecting the ECM. Nevertheless, further research is warranted to elucidate the mechanism involved in the regulation of NP cell apoptosis.

\section{Declarations}

\section{Ethics approval and consent to participate}

After the review by the Ethics Committee of the First Affiliated Hospital of Zhengzhou University, the research content and process of this project comply with the ethical requirements of biomedical research 
issued by the international and national governments, and the project is approved to be carried out in our hospital

\section{Consent for publication}

Not applicable

\section{Availability of data and materials}

The datasets used and analysed during the current study are available from the corresponding author on reasonable request.

\section{Competing interests}

The authors declare that they have no competing interests.

\section{Funding}

Not applicable

\section{Authors' contributions}

Authors in this manuscript have contributed the work below: Song Zhang contributes to the conception or design of the work and the acquisition,analysis, interpretation of data for the work; Xin-chang Lu and Tong-yu Geng contribute to the experiments operation; Heng-tao Tang contributes to draft the work and revise it critically for important intellectual content the manuscript final revision.

\section{Acknowledgements}

Not applicable.

\section{Ethical statement}

The present study has been approved by the ethics committee of the First Affiliated Hospital of Zhengzhou University. All animal experiments in this study were carried out in accordance with the National Institutes of Health guide for the care and use of Laboratory animals (NIH Publications No. 8023, revised 1978).

\section{References}

[1] Sun Z, Liu B, Luo ZJ. The Immune Privilege of the Intervertebral Disc: Implications for Intervertebral Disc Degeneration Treatment. INT J MED SCI 2020;5:685-92.

[2] Li HR, Cui Q, Dong ZY, Zhang JH, Li HQ, Zhao L. Downregulation of miR-27b is Involved in Loss of Type II Collagen by Directly Targeting Matrix Metalloproteinase 13 (MMP13) in Human Intervertebral Disc 
Degeneration. Spine (Phila Pa 1976) 2016;3:E116-23.

[3] Li Z, Chen X, Xu D, Li S, Chan M, Wu W. Circular RNAs in nucleus pulposus cell function and intervertebral disc degeneration. Cell Prolif 2019;6:e12704.

[4] Lv F, Leung VY, Huang S, Huang Y, Sun Y, Cheung KM. In search of nucleus pulposus-specific molecular markers. Rheumatology (Oxford) 2014;4:600-10.

[5] Hua WB, Wu XH, Zhang YK, et al. Dysregulated miR-127-5p contributes to type II collagen degradation by targeting matrix metalloproteinase-13 in human intervertebral disc degeneration. BIOCHIMIE 20177480.

[6] Cao Z, Chen L. Inhibition of miR-27a suppresses the inflammatory response via the p38/MAPK pathway in intervertebral disc cells. EXP THER MED 2017;5:4572-8.

[7] Liu G, Cao P, Chen H, Yuan W, Wang J, Tang X. MiR-27a regulates apoptosis in nucleus pulposus cells by targeting PI3K. PLOS ONE 2013;9:e75251.

[8] Miyazawa K, Miyazono K. Regulation of TGF-beta Family Signaling by Inhibitory Smads. Cold Spring Harb Perspect Biol 2017;3.

[9] Sedlmeier G, Sleeman JP. Extracellular regulation of BMP signaling: welcome to the matrix. Biochem Soc Trans 2017;1:173-81.

[10] Zhu J, Zhang X, Gao W, Hu H, Wang X, Hao D. IncRNA/circRNAmiRNAmRNA ceRNA network in lumbar intervertebral disc degeneration. MOL MED REP 2019;4:3160-74.

[11] Su C, Huang DP, Liu JW, Liu WY, Cao YO. miR-27a-3p regulates proliferation and apoptosis of colon cancer cells by potentially targeting BTG1. ONCOL LETT 2019;3:2825-34.

[12] Li Z, Chen X, Xu D, Li S, Chan M, Wu W. Circular RNAs in nucleus pulposus cell function and intervertebral disc degeneration. Cell Prolif 2019;6:e12704.

[13] Penolazzi L, Lambertini E, Scussel BL, et al. Reciprocal Regulation of TRPS1 and miR-221 in Intervertebral Disc Cells. CELLS-BASEL 2019;10.

[14] Hamamura K, Lin CC, Yokota H. Salubrinal reduces expression and activity of MMP13 in chondrocytes. Osteoarthritis Cartilage 2013;5:764-72.

[15] Lian C, Gao B, Wu Z, et al. Collagen type II is downregulated in the degenerative nucleus pulposus and contributes to the degeneration and apoptosis of human nucleus pulposus cells. MOL MED REP 2017;4:4730-6.

[16] Cai C, Min S, Yan B, et al. MiR-27a promotes the autophagy and apoptosis of IL-1beta treatedarticular chondrocytes in osteoarthritis through PI3K/AKT/mTOR signaling. Aging (Albany NY) 
[17] Wang Y, Cao L, Wang Q, Huang J, Xu S. LncRNA FOXD2-AS1 induces chondrocyte proliferation through sponging miR-27a-3p in osteoarthritis. Artif Cells Nanomed Biotechnol 2019;1:1241-7.

[18] Liu L, Qian H, Hu K, et al. [miR-27a-3p inhibits pulmonary fibrosis by blocking Wnt3a/beta-catenin pathway in rats]. Xi Bao Yu Fen Zi Mian Yi Xue Za Zhi 2018;11:1015-20.

[19] Zhuang YS, Liao YY, Liu BY, et al. MicroRNA-27a mediates the Wnt/beta-catenin pathway to affect the myocardial fibrosis in rats with chronic heart failure. CARDIOVASC THER 2018e12468.

[20] Wu J, Liu S, Yuan ZW, et al. MicroRNA-27a Suppresses Detrusor Fibrosis in Streptozotocin-Induced Diabetic Rats by Targeting PRKAA2 Through the TGF-beta1/Smad3 Signaling Pathway. CELL PHYSIOL BIOCHEM 2018;4:1333-49.

[21] Li X, Xu M, Ding L, Tang J. MiR-27a: A Novel Biomarker and Potential Therapeutic Target in Tumors. J CANCER 2019;12:2836-48.

[22] Yan X, Yu H, Liu Y, Hou J, Yang Q, Zhao Y. miR-27a-3p Functions as a Tumor Suppressor and Regulates Non-Small Cell Lung Cancer Cell Proliferation via Targeting HOXB8. Technol Cancer Res Treat 20191078129619.

[23] Hu HH, Chen DQ, Wang YN, et al. New insights into TGF-beta/Smad signaling in tissue fibrosis. Chem Biol Interact 201876-83.

[24] Kuo SJ, Yang WH, Liu SC, Tsai CH, Hsu HC, Tang CH. Transforming growth factor beta1 enhances heme oxygenase 1 expression in human synovial fibroblasts by inhibiting microRNA 519b synthesis. PLOS ONE 2017;4:e176052.

[25] Li X, Guo L, Yang X, et al. TGF-beta1-induced connexin43 promotes scar formation via the Erk/MMP1/collagen III pathway. J ORAL REHABIL 2019.

[26] Barruet E, Morales BM, Lwin W, et al. The ACVR1 R206H mutation found in fibrodysplasia ossificans progressiva increases human induced pluripotent stem cell-derived endothelial cell formation and collagen production through BMP-mediated SMAD1/5/8 signaling. STEM CELL RES THER 2016;1:115.

[27] Li Y, Li Z, Li C, Zeng Y, Liu Y. Long noncoding RNA TM1P3 is involved in osteoarthritis by mediating chondrocyte extracellular matrix degradation. J CELL BIOCHEM 2019;8:12702-12.

[28] Hodgkinson T, Wignall F, Hoyland JA, Richardson SM. High BMPR2 expression leads to enhanced SMAD1/5/8 signalling and GDF6 responsiveness in human adipose-derived stem cells: implications for stem cell therapies for intervertebral disc degeneration. J TISSUE ENG 20201542318554. 
[29] Wang Z, Fu C, Chen Y, et al. FoxC2 Enhances BMP7-Mediated Anabolism in Nucleus Pulposus Cells of the Intervertebral Disc. PLOS ONE 2016;1:e147764.

\section{Figures}

A

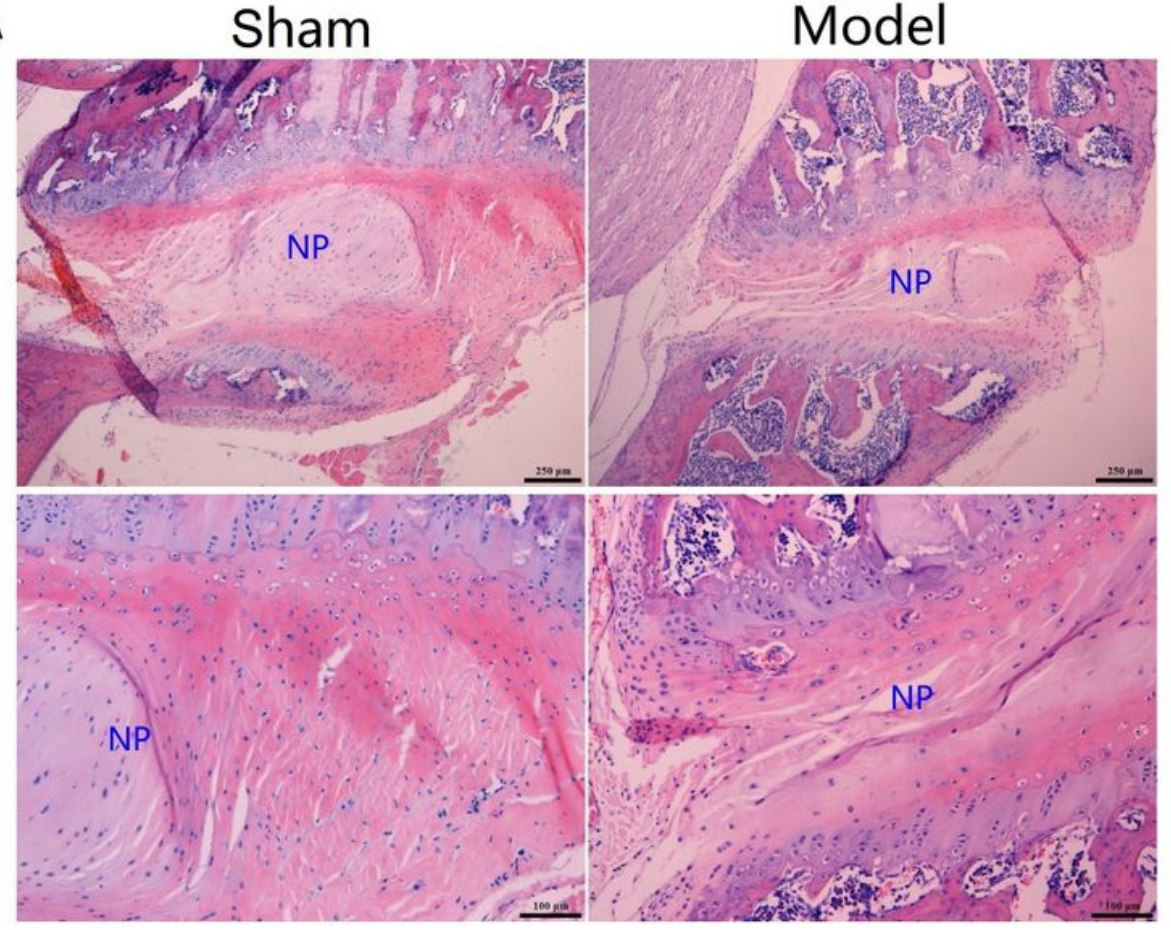

B

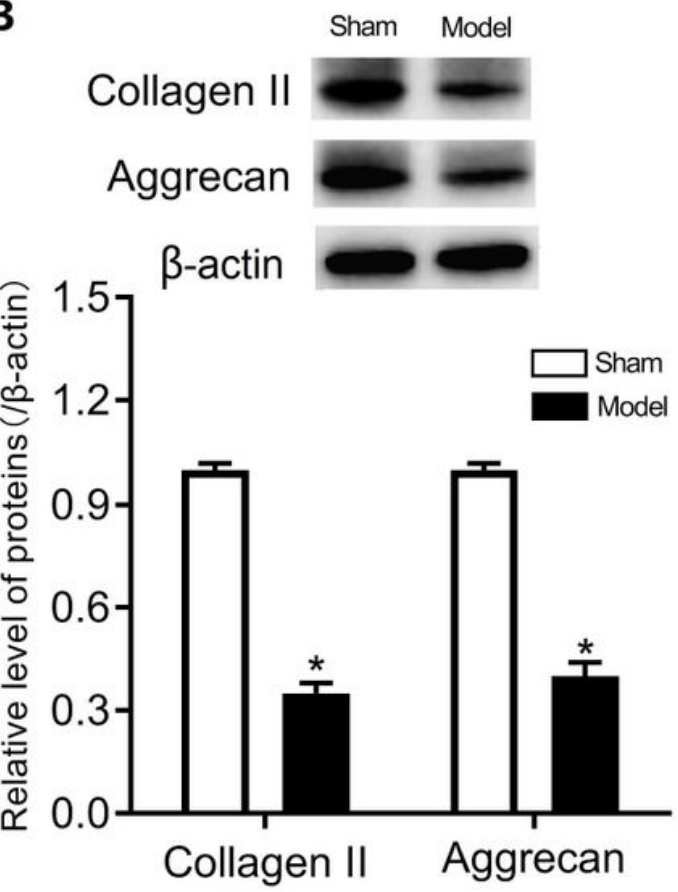

Figure 1

IDD model identification A: Pathological observation (HE staining), NP: NP. B: Expression of Collagen II and Aggrecan in IDD tissues. Compared to the sham group, ${ }^{*} \mathrm{P}<0.05$. 

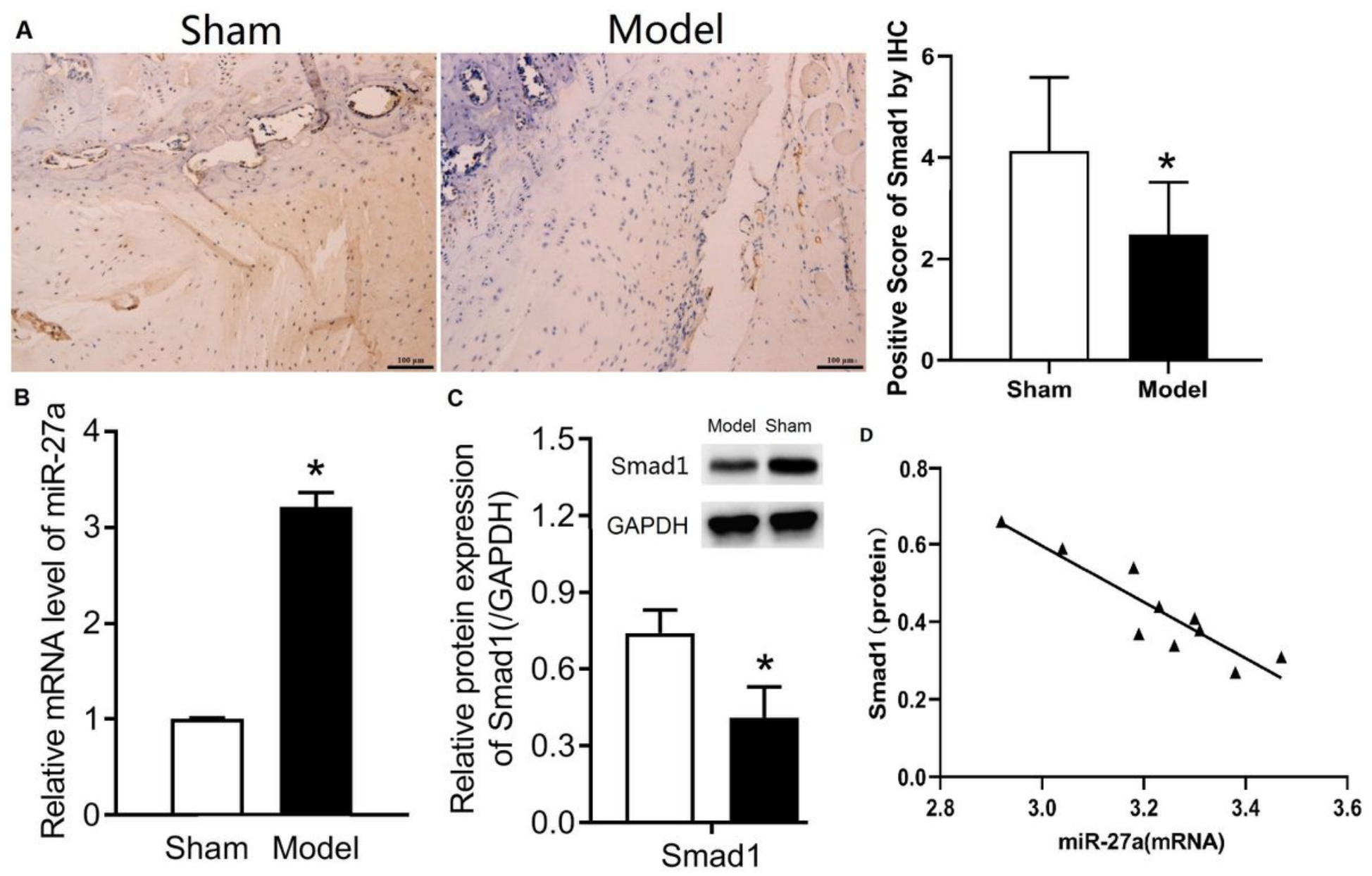

Figure 2

Expression of miR-27a and Smad1 in IDD model A:Expression of Smad1 in IDD model was detected by IHC; B: Expression of miR-27a in IDD model; C: Expression of Smad1 in IDD model was detected by western blot. D: The correlation of miR-27a and Smad1. Compared to the sham group, ${ }^{*} \mathrm{P}<0.05$. 
A

OGD
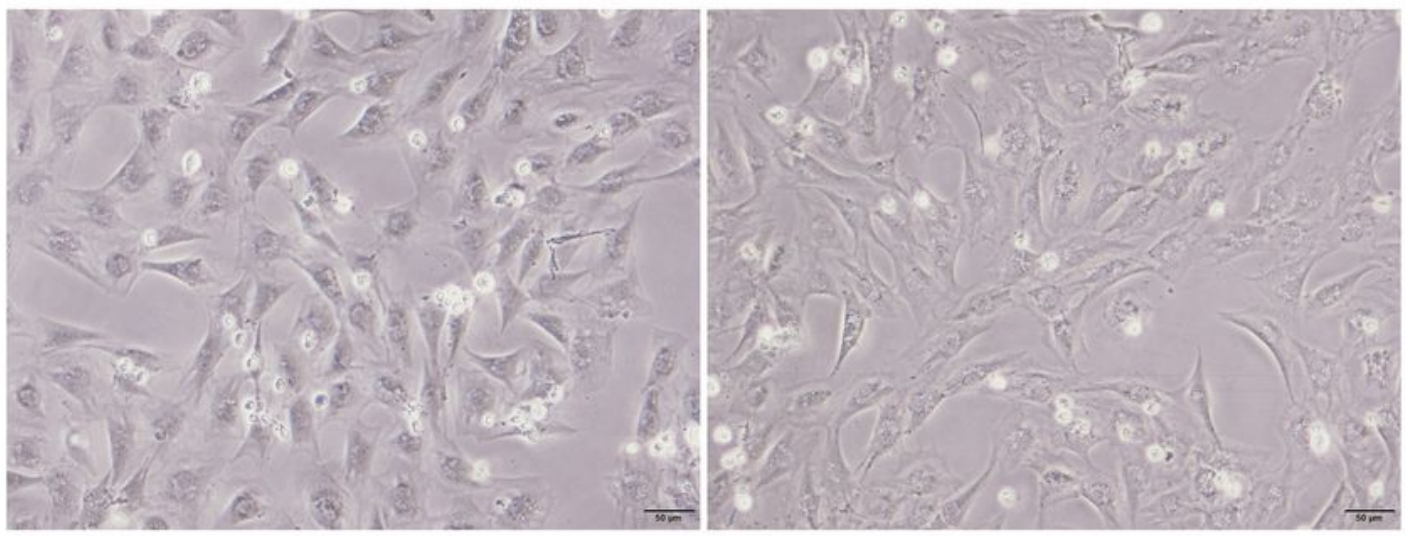

inhibitor-NC

miR-27a inhibitor
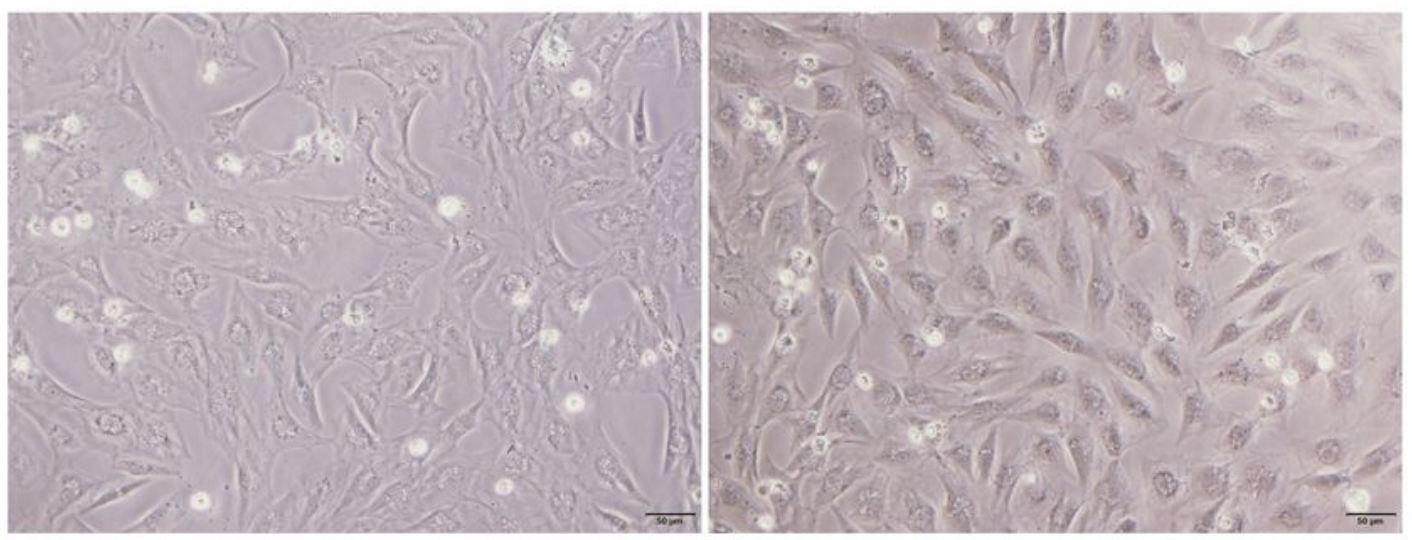

B
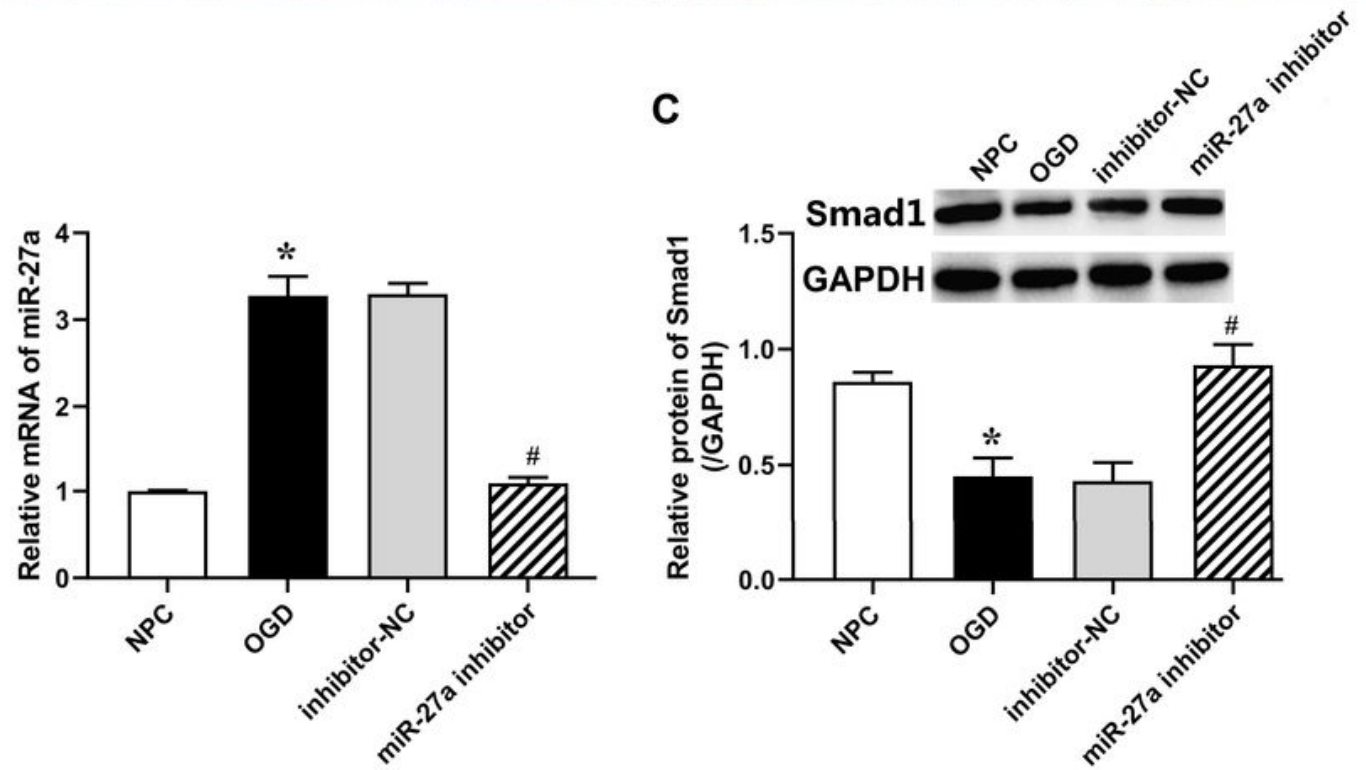

Figure 3

The expression of miR-27a and Smad1 in OGD NP cells A: The cell morphology was observed under the light microscope. B: Expression of miR-27a in NP cells. C: Expression of Smad1 in NP cells was detected by Western blot. Compared to the NPC group, ${ }^{\star} \mathrm{P}<0.05$; compared to the OGD group, $\# \mathrm{P}<0.05$. 
A

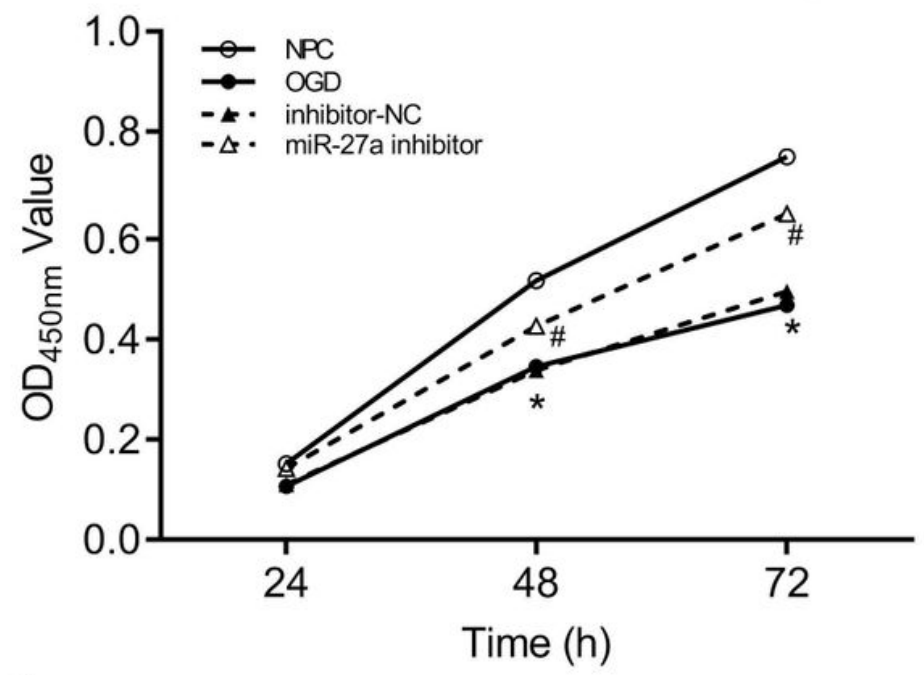

C

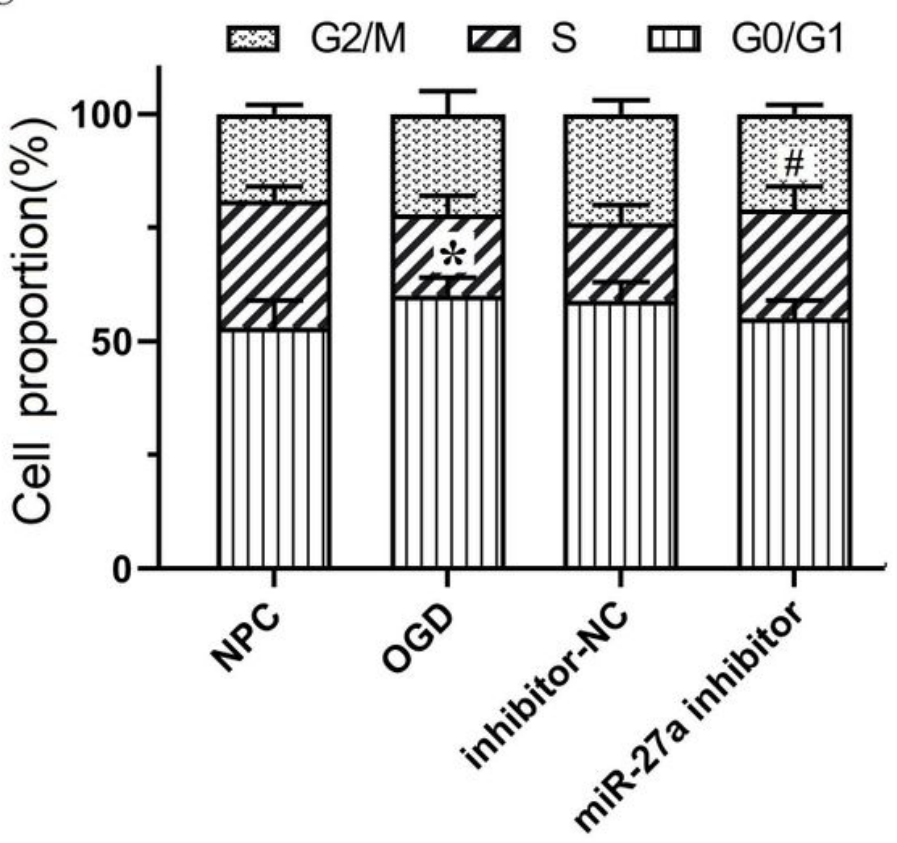

B
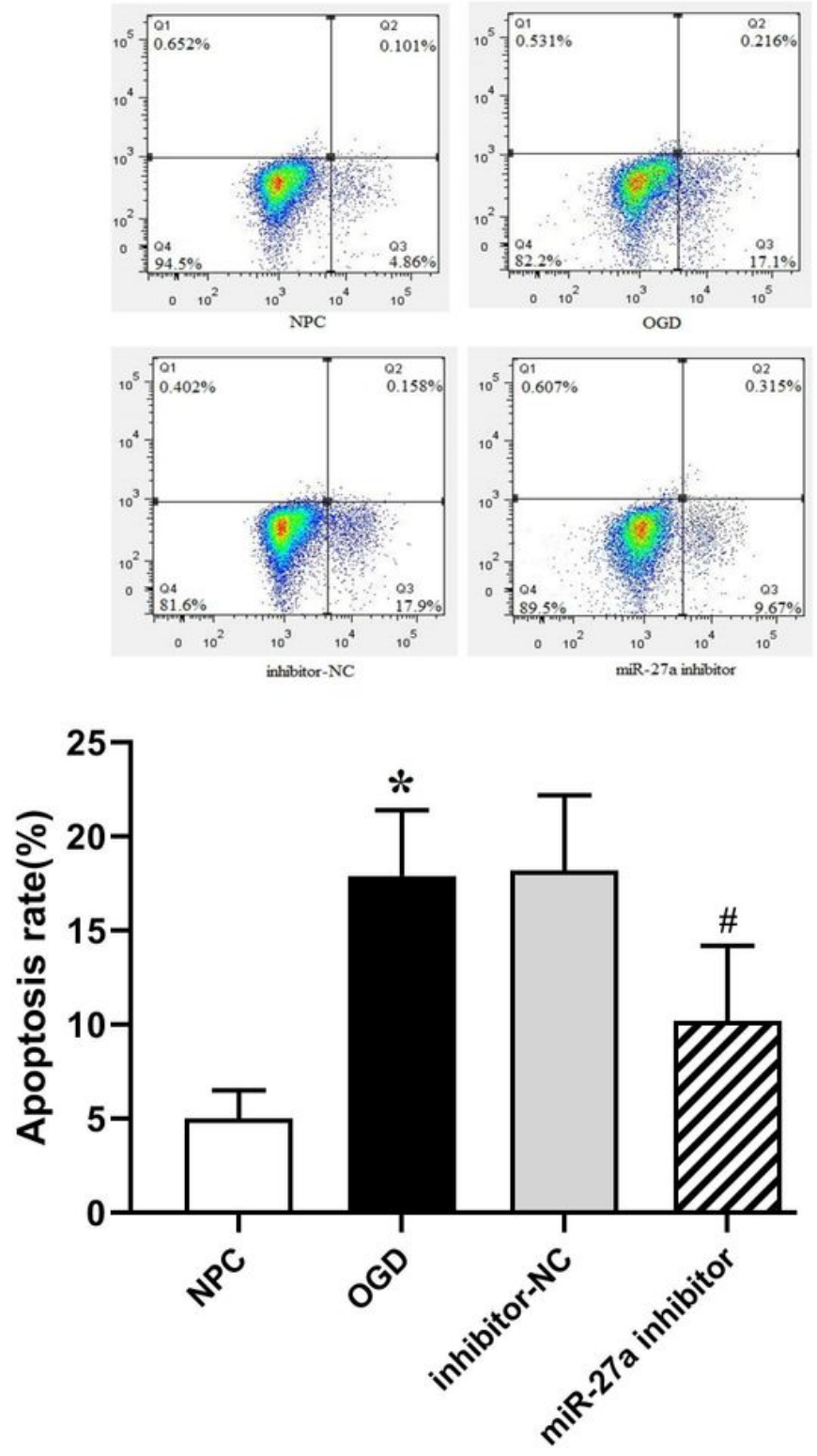

Figure 4

Down-regulation of miR-27a promoted proliferation and inhibits apoptosis of OGD-NP cells. A: Effect of down-regulation of miR-27a on proliferation of OGD-NP cells. B: Effect of down-regulation of miR-27a on apoptosis of OGD-NP cells. C: Effect of down-regulation of miR-27a on OGD-NP cell cycle. Compared to the NPC group, ${ }^{*} \times 0.05$; compared to the OGD group, $\# P<0.05$. 


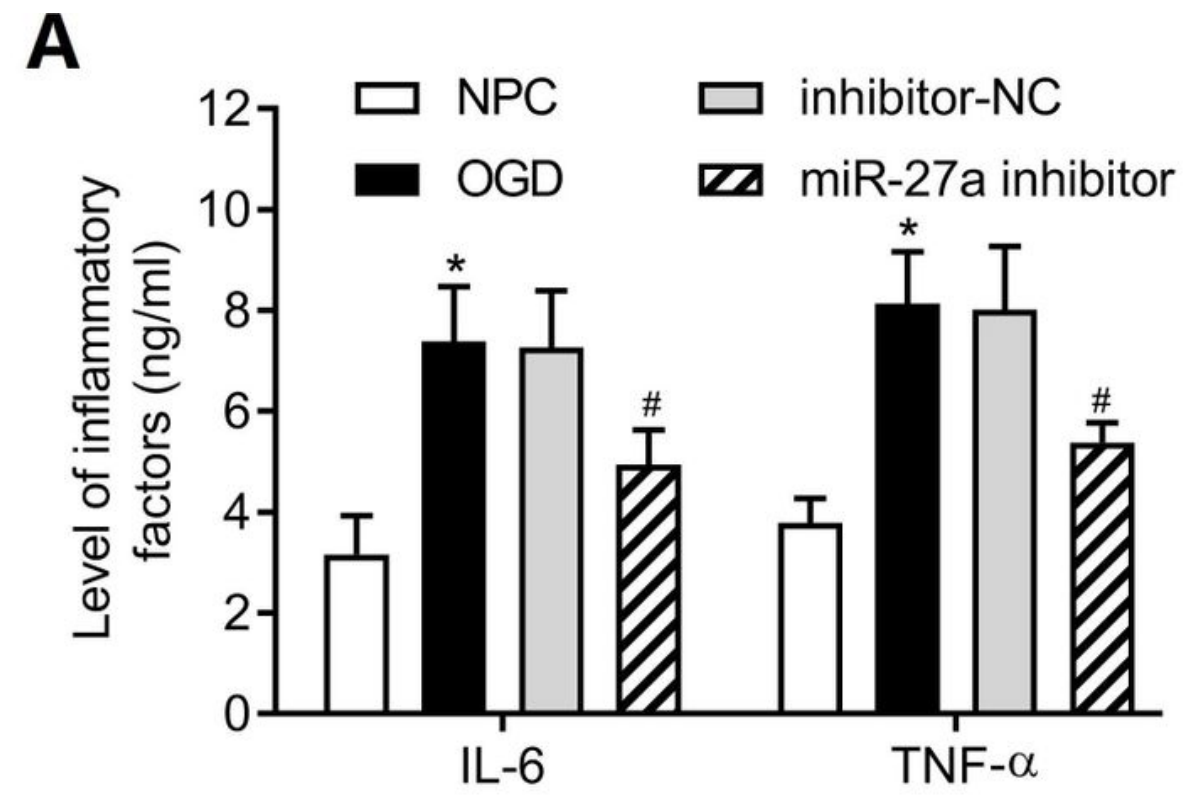

B
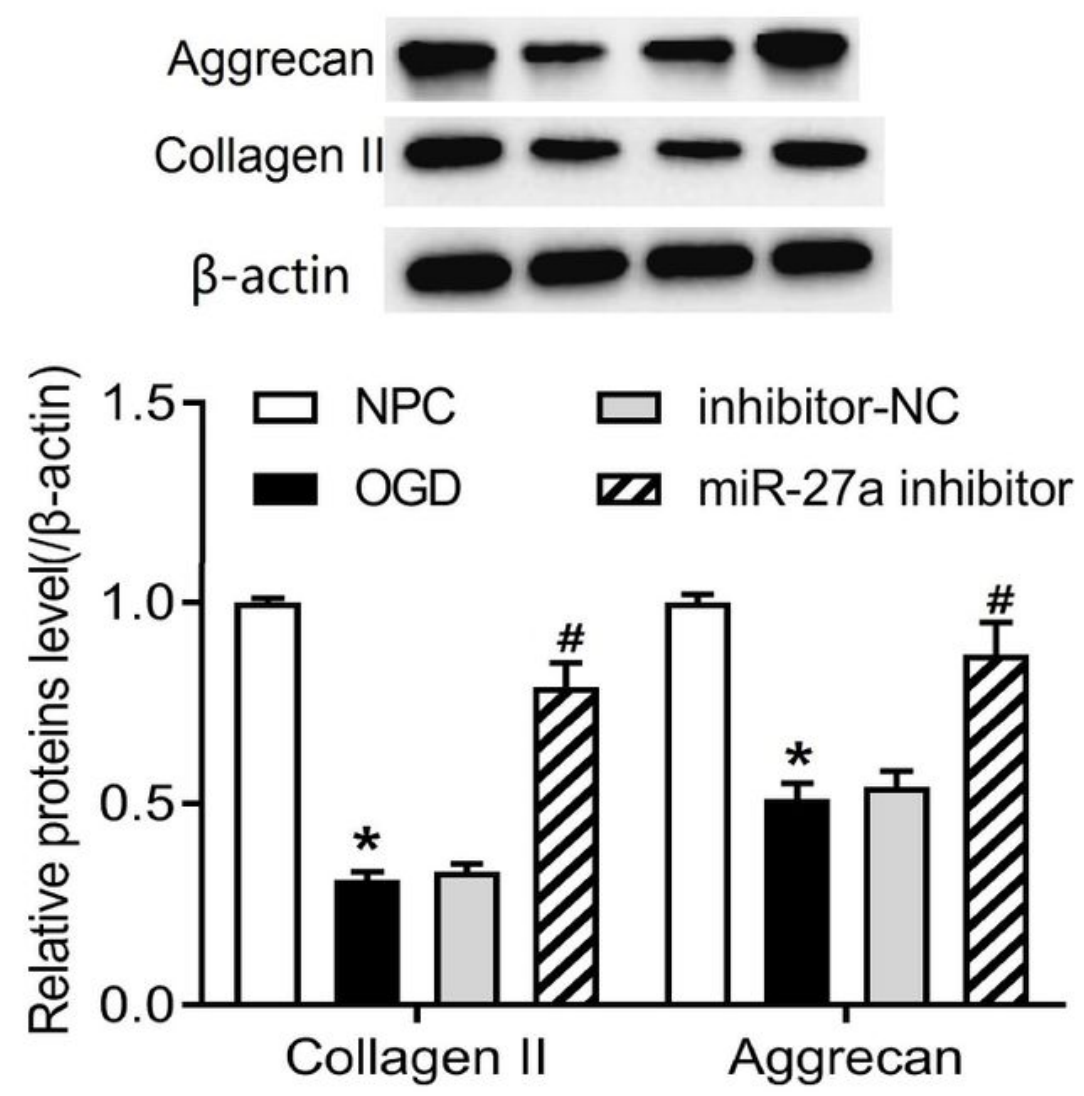

Figure 5

Overexpression of miR-27a reduces the level of inflammatory factors and the degradation of extracellular matrix. A: Overexpression of miR-27a reduces the level of inflammatory factors. B: Overexpression of miR-27a reduces the degradation of extracellular matrix. compared to the NPC group, ${ }^{\star} \mathrm{P}<0.05$; Compared to the OGD group, $\# \mathrm{P}<0.05$. 
A

Smad1 Target: 5' auaauacuvGaccucugugac 3'

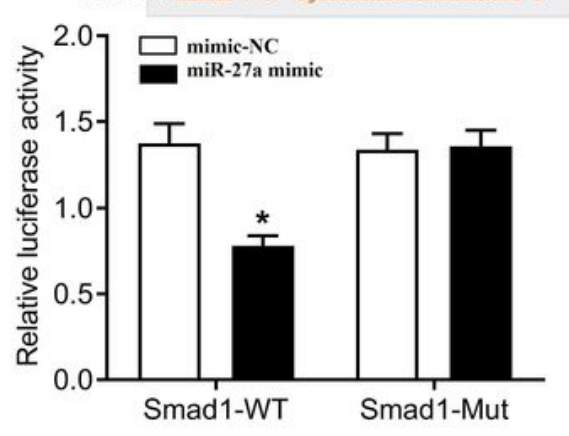

B

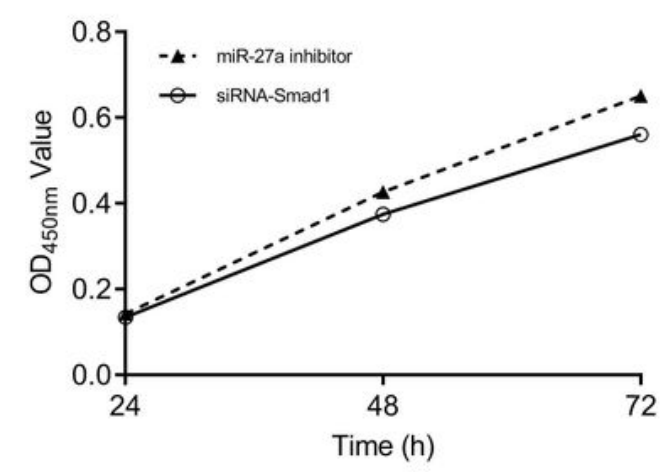

C
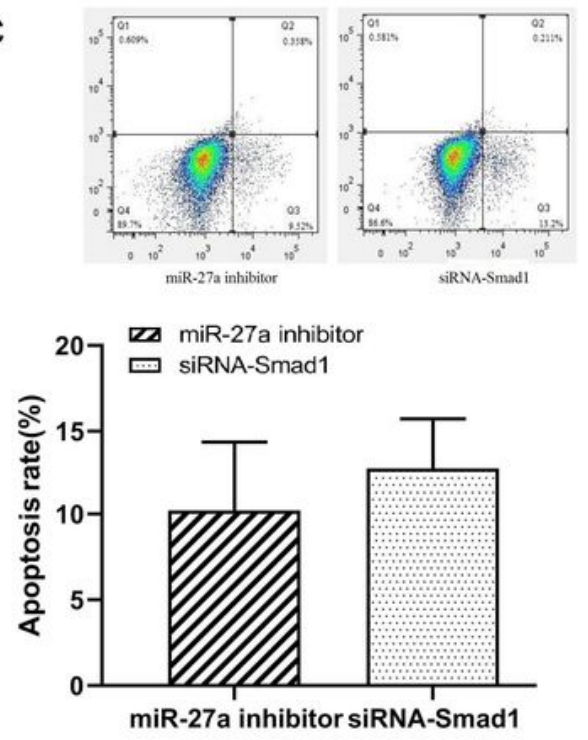

D

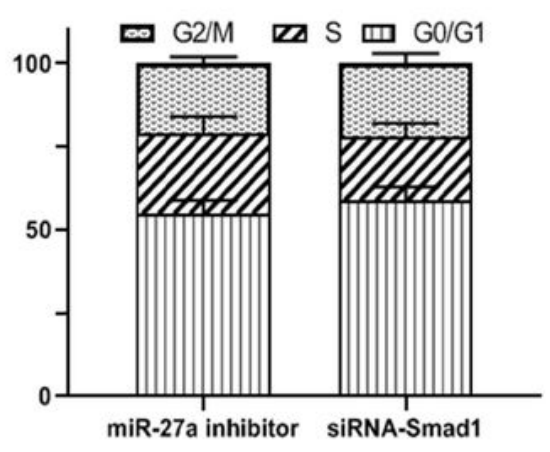

E

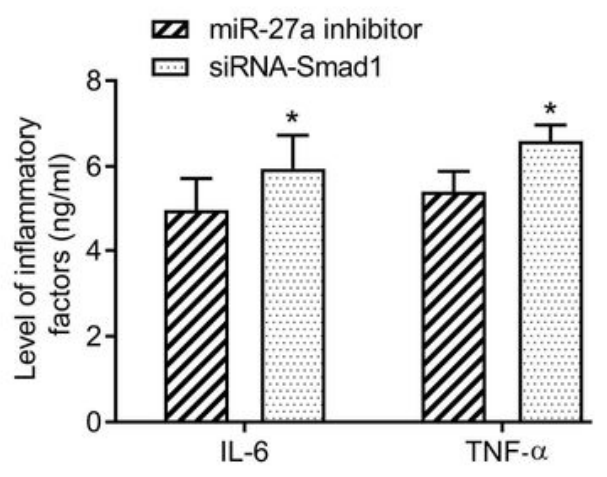

$\mathrm{F}$
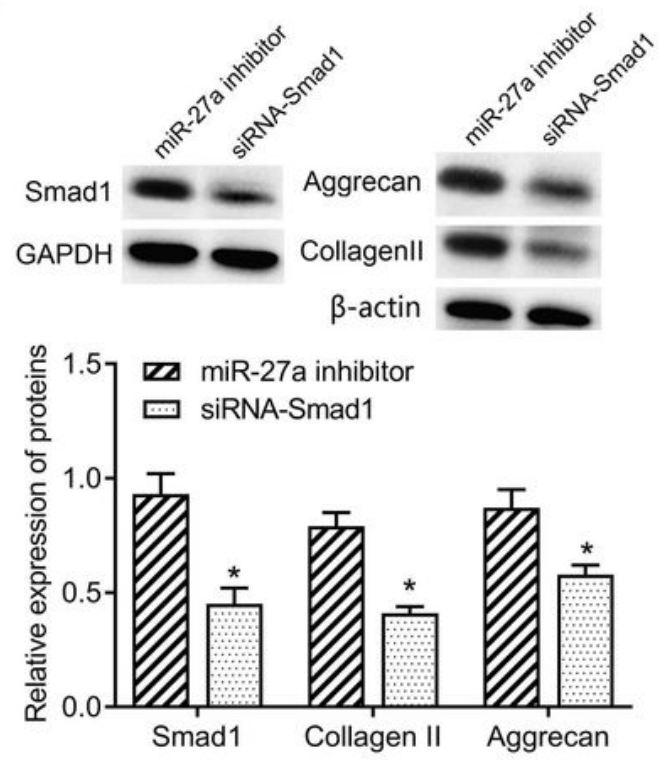

\section{Figure 6}

miR-27a targets Smad1 to regulate OGD-NP cells. A:The targeting relationship between miR-27a and smad1; compared to the miR-27a mimic+Smad1-Mut group, ${ }^{*}<0.05$. B: Effect of miR-27a inhibitor cotransfection with siRNA-Smad1 on cell proliferation ability. C: Effect of miR-27a inhibitor co-transfection with siRNA-Smad1 on cell apoptosis. D: Effect of miR-27a inhibitor co-transfection with siRNA-Smad1 on cell cycle. E: Effect of miR-27a inhibitor co-transfection with siRNA-Smad1 on inflammatory factor. F: 
Effect of miR-27a inhibitor co-transfection with siRNA-Smad1 on the degradation of extracellular matrix. Compared to the mir-27a inhibitor group, ${ }^{*} \mathrm{P}<0.05$. 